\title{
The message delay in mobile ad hoc networks
}

\author{
Robin Groenevelt ${ }^{\mathrm{a}, \mathrm{b}, *}$, Philippe Nain ${ }^{\mathrm{a}}$, Ger Koole $^{\mathrm{c}}$ \\ a INRIA, BP 93, 06902 Sophia Antipolis, France \\ ${ }^{\mathrm{b}}$ Université de Nice, Sophia Antipolis, France \\ ${ }^{c}$ Vrije Universiteit, Boelelaan 1081a, 1081 HV Amsterdam, The Netherlands
}

Available online 15 August 2005

\begin{abstract}
A stochastic model is introduced that accurately models the message delay in mobile ad hoc networks where nodes relay messages and the networks are sparsely populated. The model has only two input parameters: the number of nodes and the parameter of an exponential distribution which describes the time until two random mobiles come within communication range of one another. Closed-form expressions are obtained for the Laplace-Stieltjes transform of the message delay, defined as the time needed to transfer a message between a source and a destination. From this we derive both a closed-form expression and an asymptotic approximation (as a function of the number of nodes) of the expected message delay. As an additional result, the probability distribution function is obtained for the number of copies of the message at the time the message is delivered. These calculations are carried out for two protocols: the two-hop multicopy and the unrestricted multicopy protocols. It is shown that despite its simplicity, the model accurately predicts the message delay for both relay strategies for a number of mobility models (the random waypoint, random direction and the random walker mobility models).
\end{abstract}

(c) 2005 Elsevier B.V. All rights reserved.

Keywords: Mobile ad hoc network; Routing protocol; Absorbing Markov chain; Mobility model; Stochastic process; Message delay

\footnotetext{
* Corresponding author.

E-mail address: robin.groenevelt@ sophia.inria.fr (R. Groenevelt ).
} 


\section{Introduction}

In mobile ad hoc networks (MANET), a mobile (or simply a node) can only send data to another node if both nodes are within transmission range of one another or in contact. Two nodes are within transmission range of one another if the distance between them does not exceed $r$.

The fact that two nodes are in contact is of course not enough to ensure the success of a transmission, since many phenomena may occur during the transmission and cause it to fail (interference, physical obstacles, power problems, etc.). Message relaying is a technique that reduces message latency by using intermediary nodes to forward the message.

Routing protocols using relay nodes $[9,10,17]$ have been proposed that increase the message delivery ratio in mobile ad hoc networks. These protocols operate on a store-carry-forward mode to take advantage of node mobility to improve node connectivity, and ultimately the message throughput. When information is available (node movement, node position, etc.) these protocols may use it in a static [17] or in a dynamic [9] way. The concept of relay nodes can also be used when no information on the nodes is available [10].

Evaluating the performance of relay protocols (message delivery ratio, message latency, throughput, etc.) is a difficult task due to the inherent complexity of mobile ad hoc networks, particularly the random nature of both the movement of the nodes and of the demand (traffic). The performance of mobile ad hoc networks is in general studied via lengthy and complex simulations, for a limited number of mobility models, including the random waypoint mobility model [3] or the random direction mobility model [1,8].

In this paper we introduce a simple stochastic model to evaluate the performance of relay protocols for MANET. The model is generic and has only two input parameters: the number of nodes in the network and the intensity $(\lambda)$ of some identical and independent Poisson processes. In particular, the model does not require knowledge of the stationary distribution of the location of the nodes as input.

These processes model instances, called meeting times, at which any pair of nodes come within transmission range of one another. Transmissions between two nodes can only take place at meeting times and are assumed to be instantaneous. The latter assumption models the situation where the transmission time of a message is very small with respect to the inter meeting times. Therefore, the random nature of a MANET is captured in our model through a finite number of these independent and homogeneous Poisson processes.

The selection of the intensity $\lambda$ will be discussed in Sections 3 and 4 . The model is used to characterize the message delay between two arbitrary nodes - hereafter called the source node and the destination node - for two relay protocols and for three mobility models.

The two relay protocols are the two-hop multicopy and the unrestricted multicopy protocol. In the two-hop multicopy protocol the source node copies the message to all the nodes it meets along its route, including of course the destination node. Any node which has received a duplicate copy of the message from the source node may only forward it to the destination node. Note that this is different from the two-hop relay protocol proposed in [7]: there a packet is relayed to another node (instead of being copied).

In the unrestricted multicopy protocol the source node copies the message to all the nodes it meets (as in the two-hop multicopy protocol), but in this protocol any node that carries the message may in turn copy the message to all the nodes it encounters, along its trajectory.

The three mobility models that we will consider in this paper are the random waypoint, the random direction, and the two-dimensional random walker mobility model. All three models and their mathematical properties will be described in Section 3.1. 
The characterization of the message delay in MANET has received some attention, although explicit expressions are seldom obtained for two-dimensional mobility models. In [14] it is shown that, under the two-hop relay protocol, the expected message delay is of the order $n T_{\mathrm{p}}(n)$ for the random waypoint mobility model on a sphere (where $n$ is the number of nodes per unit area and $T_{\mathrm{p}}(n)$ is the transmission time of a message). With nodes moving as independent Brownian motions on a sphere, it is shown in [15] that the expected message delay is of the order $\log ^{2}(n) / \sigma^{2}$, where $\sigma^{2}$ is the variance parameter of the Brownian motion. In [6] the expected message delay under the unrestricted multicopy protocol is computed for a uni-dimensional network topology, where the nodes move in adjacent segments according to independent and reflected Brownian motions.

The paper is organized as follows: the stochastic model is introduced in Section 2.1, then we compute in Section 2.2 the Laplace-Stieltjes transform (LST) of the message delay (Proposition 1). In this proposition, we also obtain the distribution of the number of copies of the message at the time the message is delivered to the destination node. In Proposition 2 we calculate the expected message delay in closed-form and also find an asymptotic for a large number of nodes. These calculations are done for the two relay protocols.

In Section 3, the expected message delay and the distribution of the number of copies of the message found in Section 2 are compared to results obtained by simulations. The simulations have been carried out for each of the six combinations of the two relay protocols and the three mobility models. The simulation results are very close to the analytical results. We observed discrepancies only when the node transmission range is large with respect to the size of the area in which the nodes move. In addition, an explicit expression is given for the parameter $\lambda$ for the random waypoint and random direction mobility models, and it is shown that it hebaves as a linear function of the transmission range.

The model assumptions have been validated in Section 3 in the absence of interference (a situation that will typically occur when the node density is small and the communication radius of the nodes is small with respect to the area in which the nodes move). One way to incorporate interference into our model is to thin the meeting time sequences: with some probability $p$ (resp. $1-p$ ) a transmission occurring at a meeting time will be a success (resp. failure). Due to the fact that a thinned Poisson process is again a Poisson process, it is enough to replace $\lambda$ by $\lambda p$, with $p$ the probability that a communication fails due to interferences. We will not pursue the derivation of the value of $p$.

On the other hand, we may also argue that the communication radius of the nodes must be small enough so that interferences remain at an acceptable level. It has been shown in [7] that the transmission range of the nodes should be of the order $1 / \sqrt{N}$ for the two-hop relay protocol, in order to maintain a constant capacity per node (with $N$ the number of nodes per unit area). In Section 4 it will be shown how our model can be used to compute the expected message delay for the two relay protocols considered in this paper when the transmission range is a decreasing function of $N$.

A word on the notation: given a function $g(N)$, we write $f(N)=\mathcal{O}(g(N))$ if $|f(N) / g(N)|$ is bounded from above as $N \rightarrow \infty$ and $f(N)=\mathrm{o}(g(N))$ if $f(N) / g(N) \rightarrow 0$ as $N \rightarrow \infty$.

\section{The stochastic model}

We consider a network with $N+1$ identical mobile nodes. There is a single message to be delivered by a source node to a destination node. Intermediary nodes can be used as relay nodes. The goal is to determine the distribution of the message delay and the distribution of the number of copies of the message at the time the message is delivered to the destination node. 
We first introduce the model; then we use it in Section 2.2 to evaluate the performance of the two-hop multicopy and the unrestricted multicopy protocols.

\subsection{Definition of the model}

An analytical model that would carefully take into account the main features of a MANET (transmission range, mobility pattern, interferences, fading, etc.) would be mathematically intractable. Instead, we propose a model where the impact of these features are captured through a single parameter $\lambda$.

Let $0 \leq t_{i, j}(1)<t_{i, j}(2)<\cdots$ be the successive meeting times between nodes $i$ and $j(i \neq j)$. Define $\tau_{i, j}(n):=t_{i, j}(n+1)-t_{i, j}(n)$, the $n$-th inter-meeting time between nodes $i$ and $j$.

Transmissions between two nodes may only take place at meeting times and are assumed to be instantaneous. The latter assumption corresponds to the situation where the transmission time of a message between two nodes is negligible with respect to the time it takes the two nodes to meet one another (this is the case when the transmission radius is small with respect to the size of the area).

We assume that if a transmission takes place between node $i$ and $j$ (at some meeting time $t_{i, j}(n)$ ), then it will be successful. Assume that node $i$ carries the message just before time $t_{i, j}(n)$. Under the two-hop multicopy protocol node $i$ will transmit (a copy of) the message to node $j$ at time $t_{i, j}(n)$ if $i$ is the source node or if $j$ is the destination node. Under the unrestricted multicopy protocol node $i$ will always transmit the message to node $j$ at time $t_{i, j}(n)$.

Throughout the paper the following assumption will be made:

(A) the processes $\left\{t_{i, j}(n), n \geq 1\right\}, 1 \leq i, j \leq N+1, i \neq j$, are mutually independent and homogeneous Poisson processes with rate ${ }^{1} \lambda>0$. Equivalently stated, the random variables (rvs) $\left\{\tau_{i, j}(n)\right\}_{i, j, n}$ are mutually independent and exponentially distributed with mean $1 / \lambda$.

We introduce:

- $T_{2}$ (resp. $T_{\mathrm{U}}$ ), the message delay under the two-hop (resp. unrestricted) multicopy protocol, defined as the time needed to send the message (or a copy of the message) from the source to the destination;

- $N_{2} \in\{1,2, \ldots, N\}$ (resp. $N_{\mathrm{U}} \in\{1,2, \ldots, N\}$ ), the number of duplicate copies of the message in the network (excluding the original message but including the message at the destination node) at the time the message is delivered to the destination node.

For $\theta \geq 0$, let $T_{2}^{*}(\theta):=E\left[\mathrm{e}^{-\theta T_{2}}\right]$ and $T_{U}^{*}(\theta):=E\left[\mathrm{e}^{-\theta T_{\mathrm{U}}}\right]$ be the LST of $T_{2}$ and $T_{\mathrm{U}}$, respectively.

\subsection{Performance of relay protocols}

Proposition 1 gives, for each relay protocol, the LST of the message delay and the distribution of the number of copies.

\footnotetext{
${ }^{1}$ Without restrictions we can let $\lambda$ depend on the number of nodes in the network. This is discussed in Section 4.
} 
Proposition 1 (LST of message delay). Under the two-hop multicopy protocol

$$
T_{2}^{*}(\theta)=\sum_{i=1}^{N} i \frac{(N-1) !}{(N-i) !}\left(\frac{\lambda}{\lambda N+\theta}\right)^{i}
$$

and

$$
P\left(N_{2}=i\right)=\frac{i}{N^{i}} \frac{(N-1) !}{(N-i) !}, \quad i=1, \ldots, N .
$$

Under the unrestricted multicopy protocol

$$
T_{U}^{*}(\theta)=\frac{1}{N} \sum_{i=1}^{N} \prod_{j=1}^{i} \frac{\lambda j(N+1-j)}{\lambda j(N+1-j)+\theta}
$$

and

$$
P\left(N_{U}=i\right)=\frac{1}{N}, \quad i=1, \ldots, N,
$$

that is, the number of copies is uniformly distributed over $\{1, \ldots, N\}$.

Proof. For both the two-hop and the unrestricted multicopy protocols the proof is based on modeling the number of copies in the network as an absorbing finite-state Markov chain. The transition rates of these Markov chains will differ for each protocol.

For each protocol the Markov chain takes its values in $\{1,2, \ldots, N+1\}$. The Markov chain is in state $i=1,2, \ldots, N$ when there are $i$ copies of the message in the network including the original message, and it is in state $N+1$ when the message has been delivered to the destination node. Note that states $1,2, \ldots, N$ are transient states and $N+1$ is an absorbing state.

We provide a separate proof for equations (1)-(2) and (3)-(4).

The transition diagram of the Markov chain corresponding to the two-hop multicopy protocol is given in Fig. 1. Recall that under the two-hop multicopy protocol only the source node distributes copies of the message to nodes that come within its transmission range. Therefore, when there are $i$ copies in the network, then either a new copy is sent to the $N-i$ nodes which do not have a copy yet, which occurs at the rate $\lambda(N-i)$ and triggers a transition from $i$ to $i+1$, or one of these $i$ copies reaches the destination node, which occurs at the rate $\lambda i$ and triggers a transition from $i$ to $N+1$. This explains the transition diagram in Fig. 1.

The transition from $i$ to $N+1$ occurs with the probability $i \lambda /((N-i) \lambda+i \lambda)=i / N$, and the transition from $i$ from $i+1$ occurs with the complementary probability $(N-i) \lambda /((N-i) \lambda+i \lambda)=1-i / N$.

Proof of (1) and (2)

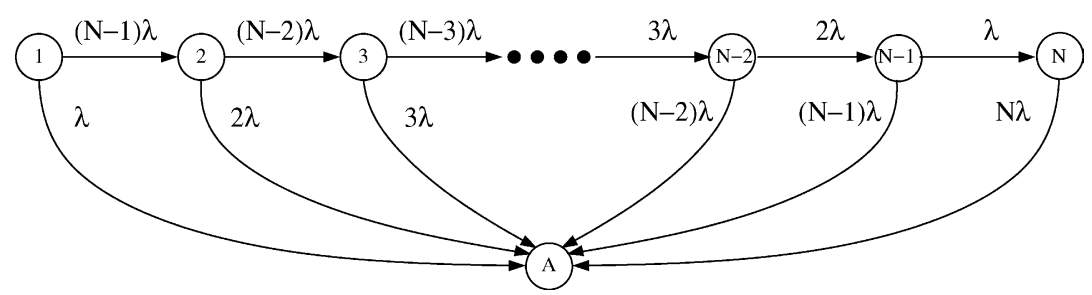

Fig. 1. Two-hop multicopy protocol: transition diagram of the Markov chain for the number of copies. 
The sojourn time $S_{i}$ in state $i=1,2, \ldots, N$ is exponentially distributed with intensity $\lambda N$ (the sum of transition rates out of state $i$ ). Moreover, $S_{1}, \ldots, S_{N}$ are mutually independent random variables.

By conditioning on the state of the Markov chain just before its enters state $N+1$, or equivalently by conditioning on the number of duplicate copies $N_{2}$ just after the message hits its destination, we have

$$
T_{2}^{*}(\theta)=\sum_{i=1}^{N} \mathbb{E}\left[\mathrm{e}^{-\theta T_{2}} \mid N_{2}=i\right] P\left(N_{2}=i\right)=\sum_{i=1}^{N} \mathbb{E}\left[\mathrm{e}^{-\theta \sum_{j=1}^{i} S_{j}} \mid N_{2}=i\right] P\left(N_{2}=i\right) .
$$

As mentioned earlier, $1-j / N$ (resp. $j / N$ ) is the probability of jumping from state $j$ to state $j+1$ (resp. $N+1)$. Therefore,

$$
P\left(N_{2}=i\right)=\frac{i}{N} \prod_{j=1}^{i-1}\left(1-\frac{j}{N}\right)=\frac{i}{N^{i}} \frac{(N-1) !}{(N-i) !},
$$

which establishes (2).

When in state $j=1,2, \ldots, N$, the Markov chain can either enter state $j+1$ after a time $S_{j, 1}$ that is exponentially distributed with intensity $(N+1-j) \lambda$, or enter state $N+1$ after a time $S_{j, 2}$, independent of $S_{j, 1}$, and exponentially distributed with intensity $j \lambda$. Observe that $S_{j}=\min \left\{S_{j, 1}, S_{j, 2}\right\}$. Moreover,

$$
P\left[S_{j, 1}<x \mid S_{j, 1}<S_{j, 2}\right]=P\left[S_{j, 2}<x \mid S_{j, 1}>S_{j, 2}\right]=P\left(S_{j}<x\right)=1-\mathrm{e}^{-\lambda N x}
$$

as a consequence of the exponential distribution. Therefore

$$
\mathbb{E}\left[\mathrm{e}^{-\theta \sum_{j=1}^{i} S_{j}} \mid N_{2}=i\right]=\mathbb{E}\left[\mathrm{e}^{-\theta\left(\sum_{j=1}^{i-1} S_{j, 1}+S_{i, 2}\right)} \mid S_{k, 1}<S_{k, 2}, k=j, \ldots, i-1, S_{i, 1}>S_{i, 2}\right] .
$$

From (7), (8) and the fact that the rvs $\left\{S_{j, k}\right\}_{j=1, \ldots, N, k=1,2}$ are mutually independent, we readily find

$$
\mathbb{E}\left[\mathrm{e}^{-\theta \sum_{j=1}^{i} S_{j}} \mid N_{2}=i\right]=\prod_{j=1}^{i} \mathbb{E}\left[\mathrm{e}^{-\theta S_{j}}\right]=\left(\frac{\lambda N}{\lambda N+\theta}\right)^{i} .
$$

Putting (5), (6) and (9) together yields

$$
T_{2}^{*}(\theta)=\sum_{i=1}^{N} i \frac{(N-1) !}{(N-i) !}\left(\frac{\lambda}{\lambda N+\theta}\right)^{i}
$$

which proves (1).

The transition diagram of the Markov chain associated with the unrestricted multicopy protocol is displayed in Fig. 2. Under this protocol, each node which has a copy of the message is allowed to distribute it to a node which does not have a copy and which comes within its transmission range. Therefore, when there are $i$ copies of the message in the network a new copy is created at the rate $\lambda i(N-i)$ (transition from $i$ to $i+1$ ) and one of these $i$ copies reaches the destination node at the rate $\lambda i$ (transition from $i$ to $N+1$ ), as depicted on Fig. 2.

The chain jumps from state $i$ to state $i+1$ with probability $(N-i) /(N+1-i)$ and it jumps from state $i$ to state $N+1$ with probability $1 /(N+1-i)$. The sojourn time $\tilde{S}_{i}$ in state $i$ is exponentially distributed with intensity $\lambda i(N+1-i)$ (obtained as the sum of the transition rates going out state $i$ ). 


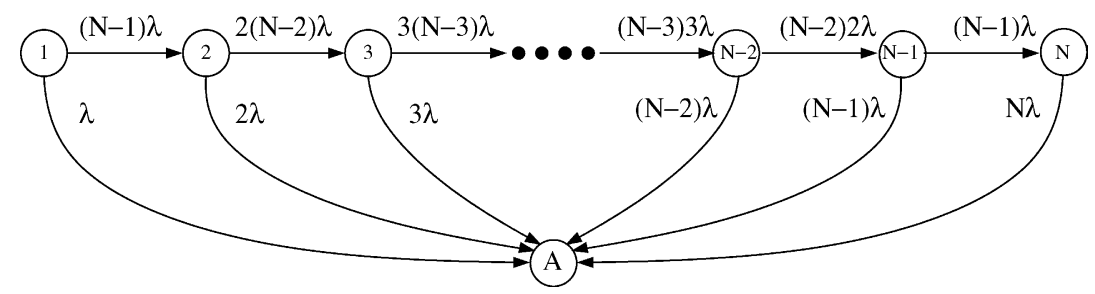

Fig. 2. Unrestricted multicopy protocol: transition diagram of the Markov chain for the number of copies.

Proof of (3) and (4)

By conditioning on the number of duplicate copies $N_{U}$, we have

$$
T_{U}^{*}(\theta)=\sum_{i=1}^{N} \mathbb{E}\left[\mathrm{e}^{-\theta \sum_{j=1}^{i} \tilde{S}_{j}} \mid N_{\mathrm{U}}=i\right] P\left(N_{\mathrm{U}}=i\right)
$$

with

$$
P\left(N_{\mathrm{U}}=i\right)=\frac{1}{N+1-i} \prod_{j=1}^{i-1} \frac{N-j}{N+1-j}=\frac{1}{N},
$$

which proves (4). Similarly to (9) we have

$$
\mathbb{E}\left[\mathrm{e}^{-\theta} \sum_{j=1}^{i} \tilde{S}_{j} \mid N_{U}=i\right]=\prod_{j=1}^{i} \mathbb{E}\left[\mathrm{e}^{-\theta \tilde{S}_{j}}\right]=\prod_{j=1}^{i} \frac{\lambda j(N+1-j)}{\lambda j(N+1-j)+\theta},
$$

which, together with equations (12) and (13), proves (3).

Proposition 2 gives an explicit expression, and an asymptotic for large $N$, for the expected message delay for each relay protocol. This result shows that for each protocol the expected message delay is a linear function of the expected inter-meeting time $1 / \lambda$, and changing the value $\lambda$ does not have any impact except for a time scaling.

Proposition 2 (Expected message delays). Under the two-hop multicopy protocol, the expected message delay is given by

$$
\mathbb{E}\left[T_{2}\right]=\frac{1}{\lambda N} \sum_{i=1}^{N} \frac{i^{2}(N-1) !}{(N-i) ! N^{i}}=\frac{1}{\lambda}\left(\sqrt{\frac{\pi}{2 N}}+\mathcal{O}\left(\frac{1}{N}\right)\right) .
$$

Under the unrestricted multicopy protocol, the expected message delay is

$$
\mathbb{E}\left[T_{\mathrm{U}}\right]=\frac{1}{\lambda N} \sum_{i=1}^{N} \frac{1}{i}=\frac{1}{\lambda N}\left(\log (N)+\gamma+\mathcal{O}\left(\frac{1}{N}\right)\right),
$$

where $\gamma \approx 0.57721$ is Euler's constant.

Proof. Since $\mathbb{E}\left[T_{2}\right]=-\left.\frac{\mathrm{d} T_{2}^{*}(\theta)}{\mathrm{d} \theta}\right|_{\theta=0}, \mathbb{E}\left[T_{2}\right]$ can be derived at once from (1). For the sake of clarity the proof of the asymptotic expansion of (12) is forwarded to Appendix A. 
Similarly, we find by differentiating (3) w.r.t. $\theta$, and then by setting $\theta=0$, that

$$
\begin{aligned}
\mathbb{E}\left[T_{\mathrm{U}}\right] & =\frac{1}{\lambda N} \sum_{i=1}^{N} \sum_{j=1}^{i} \frac{1}{j(N+1-j)}=\frac{1}{\lambda N(N+1)} \sum_{i=1}^{N} \sum_{j=1}^{i}\left(\frac{1}{j}+\frac{1}{N+1-j}\right) \\
& =\frac{1}{\lambda N(N+1)} \sum_{j=1}^{N} \sum_{i=j}^{N}\left(\frac{1}{j}+\frac{1}{N+1-j}\right)=\frac{1}{\lambda N} \sum_{j=1}^{N} \frac{1}{j},
\end{aligned}
$$

which is the first part of (13). This last summation is known as the harmonic numbers. Its asymptotic expansion [13, p.186] is $\sum_{i=1}^{N} \frac{1}{i}=\log (N)+\gamma+\mathcal{O}\left(\frac{1}{N}\right)$, where $\gamma$ is Euler's constant. This gives the second part of (13) and concludes the proof.

The second moments of the message delay can be found in [5, Section 3.2.2]. The next result gives the expected number of copies of the message at the time the message is delivered.

Corollary 3 (Expected number of copies). The expected number of copies under the two-hop multicopy protocol is given by (cf. (2) and (12))

$$
\mathbb{E}\left[N_{2}\right]=\frac{1}{N} \sum_{i=1}^{N} \frac{i^{2}}{N^{i}} \frac{N !}{(N-i) !}=\sqrt{\frac{\pi N}{2}}+\mathcal{O}(1) .
$$

Hence $\mathbb{E}\left[N_{2}\right]=\lambda N \mathbb{E}\left[T_{2}\right]$. The expected number of copies under the unrestricted multicopy protocol is (cf. (4)) $\mathbb{E}\left[N_{\mathrm{U}}\right]=\frac{N+1}{2}$.

The relative performance of the two-hop multicopy and unrestricted multicopy protocols can be captured through the ratios $\mathbb{E}\left[T_{\mathrm{U}}\right] / \mathbb{E}\left[T_{2}\right]$ and $\mathbb{E}\left[N_{\mathrm{U}}\right] / \mathbb{E}\left[N_{2}\right]$ given by (cf. Proposition 2 and Corollary 3 )

$$
\frac{\mathbb{E}\left[T_{\mathrm{U}}\right]}{\mathbb{E}\left[T_{2}\right]}=\frac{N \sum_{i=1}^{N} \frac{1}{i}}{\sum_{i=1}^{N} \frac{i^{2}}{N^{i}} \frac{N !}{(N-i) !}} \quad \text { and } \quad \frac{\mathbb{E}\left[N_{\mathrm{U}}\right]}{\mathbb{E}\left[N_{2}\right]}=\frac{N(N+1)}{2 \sum_{i=1}^{N} \frac{i^{2}}{N^{i}} \frac{N !}{(N-i) !}},
$$

respectively. Note that both ratios are independent of $\lambda$. By using the asymptotic expansions (12), (13) and (14), we see that for large $N$

$$
\frac{\mathbb{E}\left[T_{\mathrm{U}}\right]}{\mathbb{E}\left[T_{2}\right]} \approx \frac{\log (N)}{\sqrt{N}} \sqrt{\frac{2}{\pi}} \text { and } \frac{\mathbb{E}\left[N_{\mathrm{U}}\right]}{\mathbb{E}\left[N_{2}\right]} \approx \sqrt{\frac{N}{2 \pi}}
$$

For instance, if $N=10^{3}$ then $\mathbb{E}\left[T_{\mathrm{U}}\right] / \mathbb{E}\left[T_{2}\right] \approx 0.17$ and $\mathbb{E}\left[N_{\mathrm{U}}\right] / \mathbb{E}\left[N_{2}\right] \approx 12.6$.

\section{Applications}

This section is devoted to the application of the results in Section 2 to three different mobility models. It is structured as follows: the mobility models are presented in Section 3.1 and their simulation settings in Section 3.2. Through both intuitive reasoning and simulations it is shown in Section 3.3 that assumption (A) is reasonable when the transmission range is not too large relative to the surface area. Based on this observation, estimates are obtained for the meeting rate $\lambda$, for each mobility model and for various 
transmission ranges. With the help of these estimates the accuracy of the model is demonstrated in Sections 3.4 and 3.5, where the expected message delay and the distribution of the number of copies are compared to simulation results.

\subsection{Mobility models}

Although the results in Section 2 hold regardless of the dimension of the space in which the nodes move, in the following we shall only apply them to three standard two-dimensional mobility models: the random waypoint, the random direction, and the random walker mobility model.

\subsubsection{Random waypoint mobility model}

The random waypoint mobility model [3] is commonly used in the simulation of mobile ad hoc networks. In the random waypoint mobility model each node is assigned an initial location in a given area (typically a square) and travels at a constant speed $S$ to a destination chosen uniformly in this area. The speed $S$ is chosen uniformly in $\left(v_{\min }, v_{\max }\right)$, independently of the initial location and destination. After reaching the destination, the node may pause for a random amount of time after which a new destination and a new speed are chosen, independently of all previous destinations, speeds, and pause times. The stationary distributions of location and speed in the random waypoint mobility model differ significantly from the uniform distribution. In particular, it has been observed that the stationary distribution of the location of a node is more concentrated near the center of the region in which the nodes move [2]. Also, $v_{\text {min }}$ needs to be strictly positive to ensure that the average speed over time does not go to zero [16].

\subsubsection{Random direction mobility model}

In the random direction mobility model [1,8] each node is assigned an initial direction $\theta$, speed $S \in$ $\left[v_{\min }, v_{\max }\right]$ and a finite travel time $\tau$. The node then travels in the direction $\theta$ for a duration $\tau$ and at speed $S$. When the node travel time has expired a new direction, speed and travel time are chosen at random, independently of all previous directions, speeds and travel times. When a node reaches a boundary it is either reflected [1] or the area wraps around so that the node reappears on the other side [1].

The stationary distributions of the location and direction have been shown to be uniform [11] for arbitrary direction, speed and travel time distributions, irrespective of the boundaries being reflecting or wrap around. This is in contrast with the random waypoint mobility model where nodes are more likely to be concentrated near the center of the area. Another difference is that $v_{\min }$ does not have to be strictly positive. The speed can be equal to zero since the node maintains a certain speed only for a limited amount of time.

\subsubsection{Random walker mobility model}

In the two-dimensional random walker mobility model each node moves as a random walker on a two-dimensional lattice. The time is discrete and at each time step each node has a probability of $1 / 4$ of hopping to a position above, below, to the left, or to the right of its current position. If the node is positioned on a boundary, then instead of hopping off the lattice it hops back to the same state. This movement can be seen as someone wandering at a constant speed from intersection to intersection through a city, where all of the streets are equally spaced and perpendicular to each other (Manhattan network). The stationary distribution of the location of a two-dimensional random walker on a square lattice is uniform over the area. This properties is a consequence of the fact that a two-dimensional random walker can be constructed 
from two independent one-dimensional random walkers, and that the stationary location of a symmetric random walk in one-dimension is uniform (take $n \rightarrow \infty$ in formula (3.15) in [4, p. 357] to obtain the stationary distribution and then set $p=q=\frac{1}{2}$ ).

\subsection{Simulation setting}

The numerical results presented hereafter are based on simulation programs in which mobile nodes move in a square of size $4 \mathrm{~km} \times 4 \mathrm{~km}$, without pausing.

As mentioned in [12], there are several pitfalls to avoid when simulating the random waypoint mobility model. In this work we have used the implementation of the random waypoint mobility model proposed in [12] (without pausing), which consists of sampling the initial speeds and locations from their stationary distributions. Then, subsequent speeds and locations are sampled from the uniform distribution.

Since the stationary distribution of the location of a node is uniform in both the random direction mobility model and the random walker mobility model, their implementation does not pose any difficulty.

For the random waypoint mobility model and the random direction mobility model, a speed (in $\mathrm{km} / \mathrm{h}$ ) was chosen uniformly in $\left[v_{\min }, v_{\max }\right]=[4,10]$.

In the random direction mobility model, a node moves in a direction that is uniformly distributed in $[0,2 \pi$ ), for an exponential amount of time (expressed in hours) with mean $1 / 4$, and at a speed that is uniformly distributed in [4,10], before the node chooses a new direction, travel time, and speed.

For the random walker mobility model we assume the streets are $80 \mathrm{~m}$ apart and the random walkers move at the speed of one block per minute (this results in $51^{2}=2601$ states and a constant speed of $4.8 \mathrm{~km} / \mathrm{h})$.

As mentioned earlier, we assume that there is no inference and that the transmission of a message between two nodes (in contact) is instantaneous.

In order to apply the results in Section 2 we need, for each mobility model, to check the validity of assumption (A) and to identify the parameter $\lambda$ of the exponential inter-meeting time distribution.

\subsection{Validation of the Poisson meeting times}

For each mobility model and for various communication radii, we have simulated the movement of two nodes and have estimated the distribution of the inter-meeting times (Section 3.3.1) and shown the independence of the process through the autocorrelation function (Section 3.3.2). The results, based on 100,000 observations, show that the Poisson assumption for the meeting time sequences is valid for all three mobility models and for a large range of communication radii.

\subsubsection{Inter-meeting time distribution}

Fig. 3 displays, on a log-scale for the $y$-axis, the complementary cumulative distribution function (complementary cdf) of the inter-meeting time between two nodes for each mobility model and for three different communication radii $(r=50,100,250 \mathrm{~m})$.

For the random direction and the random waypoint mobility models, and for each communication radius, we have also plotted the complementary cdf of an exponential distribution (i.e. a straight line on a log-scale for the $y$ axis) with intensity (i.e. slope) $\lambda$. We observe an excellent agreement between the estimated cdf (solid line) and the exponential cdf (dashed line) for the three different communication radii. Estimates for the value of $\lambda$ for these two models can been derived and are given in Lemma 4 . The 

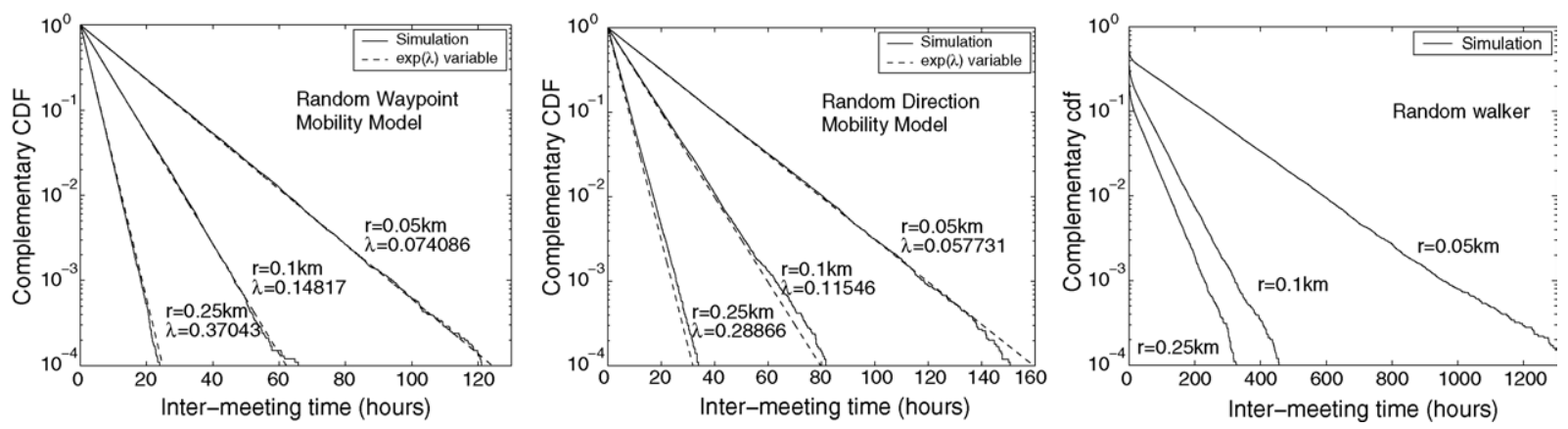

Fig. 3. Complementary cdf of the inter-meeting time of two nodes.

proof - which confirms that the meeting times can be modeled as a Poisson process - can be found in [5, Chapter 4] and has been omitted here due to space constraints.

Lemma 4 (Estimates for $\lambda$ ). The parameter $\lambda$ for the random direction $(R D)$ and the random waypoint $(R W)$ mobility models is given by

$$
\lambda_{\mathrm{RD}} \approx \frac{2 r \mathbb{E}\left[V^{*}\right]}{L^{2}}, \quad \text { and } \quad \lambda_{\mathrm{RW}} \approx \frac{2 \omega r \mathbb{E}\left[V^{*}\right]}{L^{2}},
$$

respectively, for values of $r \ll L$. Here $\omega \approx 1.3683$ is a constant specific to the random waypoint model and $\mathbb{E}\left[V^{*}\right]$ is the average relative speed between two nodes. In the special case where $v=v_{\min }=v_{\max }$ we have $\lambda_{R D} \approx \frac{8 r v}{\pi L^{2}}$ and $\lambda_{R W} \approx \frac{8 \omega r v}{\pi L^{2}}$.

The average speed $\mathbb{E}\left[V^{*}\right]$ can be calculated numerically [5, Proposition 4.2.2]. For example, if $\left[v_{\min }, v_{\max }\right]=[4,10] \mathrm{km} / \mathrm{h}$, then $\mathbb{E}\left[V_{*}\right] \approx 9.2 \mathrm{~km} / \mathrm{h}$ for the random direction and $\mathbb{E}\left[V_{*}\right] \approx 8.7 \mathrm{~km} / \mathrm{h}$ for the random waypoint mobility model. The values of $\lambda$ thus obtained are given in Figs. 3 and 4.

For the random walker mobility model the situation is more complicated: the inter-meeting time is not exponential but there is an exponential tail which rapidly emerges. The reason, we argue, is because random walkers have a tendency to "hang around" the same region (resulting in many small inter-meeting times). If, however, the two random walkers have wandered away from one another, then we find an exponential distribution for the time until they meet again. This explains why in [15] the first-meeting time (defined as the time between a random moment and the moment when two nodes meet) between two Brownian motions resembles an exponential distribution whereas the inter-meeting time does not.

Because of the quick emergence of the exponential tail for the random walker mobility, we have included it in our analysis to see how robust our model is. An explicit expression for the exponential tail under the random walker mobility model is, to the best of our knowledge, not known and it is therefore obtained numerically as the complementary of the average first-meeting time obtained across all simulations.

The fact that, for each mobility model, the cdf of the inter-meeting distribution is well-approximated by an exponential distribution, at least for small to moderate transmission radii (with respect to the size of the area) finds its roots in the various independence assumptions placed on each mobility model. Indeed, nodes move independently of each other and future directions and speeds (and therefore locations) of a node are independent of past directions and speeds of this node. If we pick two mobile nodes at random at some stationary time, then there is a probability $q$ that they will meet (in the sense of being within 


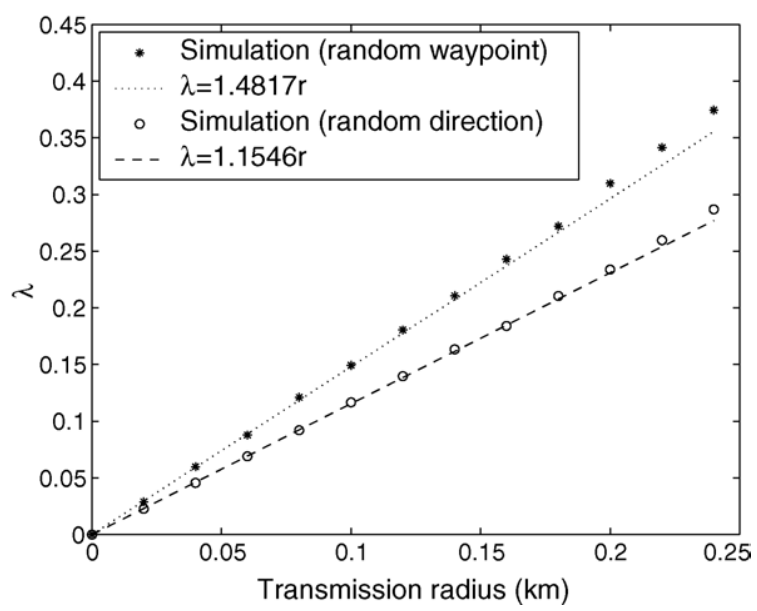

Fig. 4. Relationship between the inter-meeting time intensity $\lambda$ and the communication radius $r$.

transmission range of one another) before the next change of direction of either node. At the next change of direction, because of the independent assumptions recalled above, the process repeats itself and there is a probability $q$ that these nodes will meet before the next change of direction. This yields a geometric distribution for the number of changes of direction before both nodes meet. The exponential distribution pops up because the number of changes of direction is "linearly" related to the time traveled before the nodes meet.

\subsubsection{Independence of inter-meeting times}

Let $\{\tau(n)\}_{n}$ be the $n$-th inter-meeting times between two given nodes. To check the assumption that the rvs $\{\tau(n)\}_{n}$ are mutually independent, we have used the following classical estimator for the autocorrelation function of $\{\tau(n)\}_{n}$

$$
\rho_{m}(h)=\frac{\gamma_{m}(h)}{\gamma_{0}(h)}, \quad h \geq 0,
$$

where

$$
\gamma_{m}(h):=\frac{1}{m} \sum_{n=1}^{m-h}\left(\tau(n+h)-\hat{\tau}^{(m)}\right)\left(\tau(n)-\hat{\tau}^{(m)}\right)
$$

is an estimator of the autocovariance function, with $\hat{\tau}^{(m)}=(1 / m) \sum_{n=1}^{m} \tau(n)$ the sample mean for $m$ observations. In particular, $\rho_{0}(h)=1$.

If the rvs $\{\tau(n)\}_{n}$ are mutually independent then their autocorrelation function is equal to zero for all $h \geq 1$.

The mapping $h \rightarrow \rho_{m}(h)$ corresponding to the random waypoint mobility model is plotted in Fig. 5 for $m=100,000$ and $r=0.25 \mathrm{~km}$. The autocorrelation functions corresponding to other values of $r$ $(r=0.05 \mathrm{~km}, r=0.1 \mathrm{~km})$ and/or to the random direction mobility model and the random walker mobility model are not displayed since they are identical to the results in Fig. 5. 


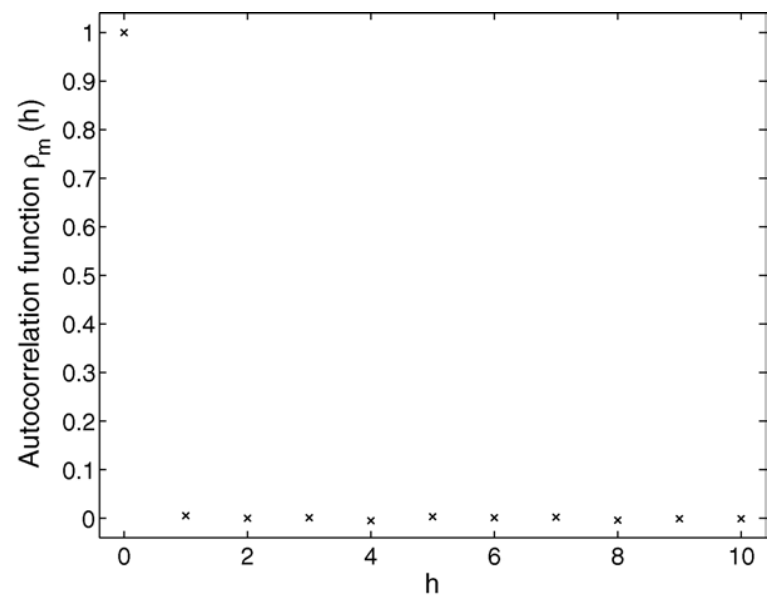

Fig. 5. Autocorrelation function of inter-meeting times for the random waypoint model with $r=0.25 \mathrm{~km}$.

Since $\rho_{m}(h)$ is very close to zero for all $h \geq 1$ we conclude that the assumption that the inter-meeting times between two nodes are mutually independent rvs is a reasonable assumption.

In conclusion, the results reported in Sections 3.3.1 and 3.3.2 validate the assumption that the meeting time process between two given nodes is a Poisson process for all three mobility models and for small to moderate communication radii (with respect to the size of the area in which the nodes move).

\subsection{Expected message delay}

For the three mobility models introduced in Section 3.1 and for three communication radii $(r=0.05$, $0.1,0.25 \mathrm{~km}$ ), Figs. 6 and 7 display the expected message delays obtained both through simulations and by the analytical model as a function of the number of nodes. Results for the two-hop (resp. unrestricted) multicopy protocol are given in Fig. 6 (resp. Fig. 7).

These results demonstrate the ability of the analytical model to predict the expected message delay under both the two-hop multicopy protocol and the unrestricted multicopy protocol for different mobility patterns, across any number of nodes and communication radii.
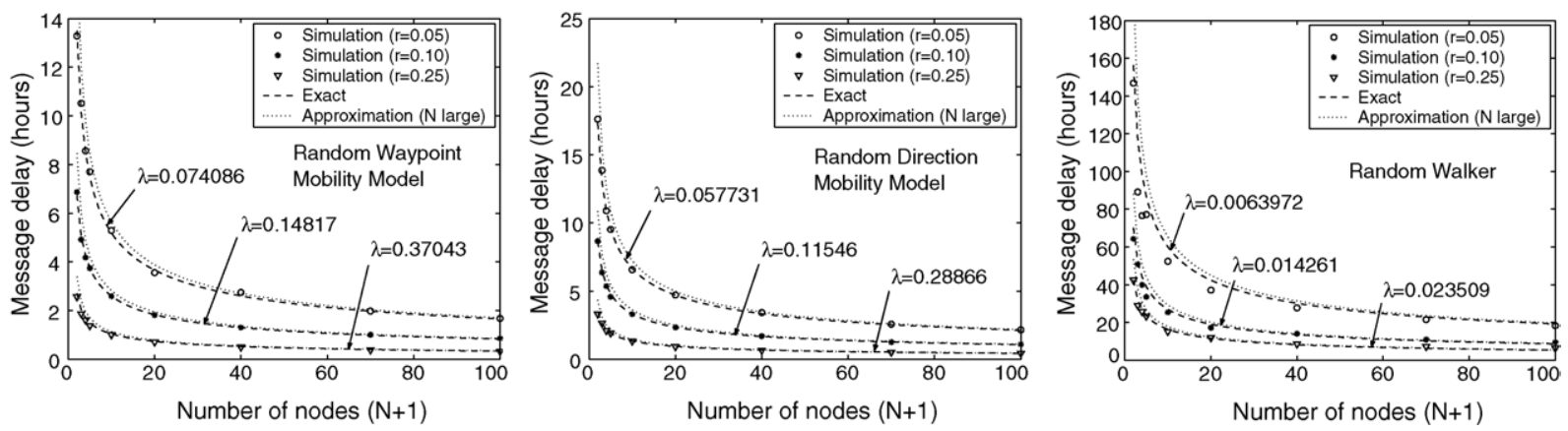

Fig. 6. Message delay vs. number of nodes: the two-hop multicopy protocol. 

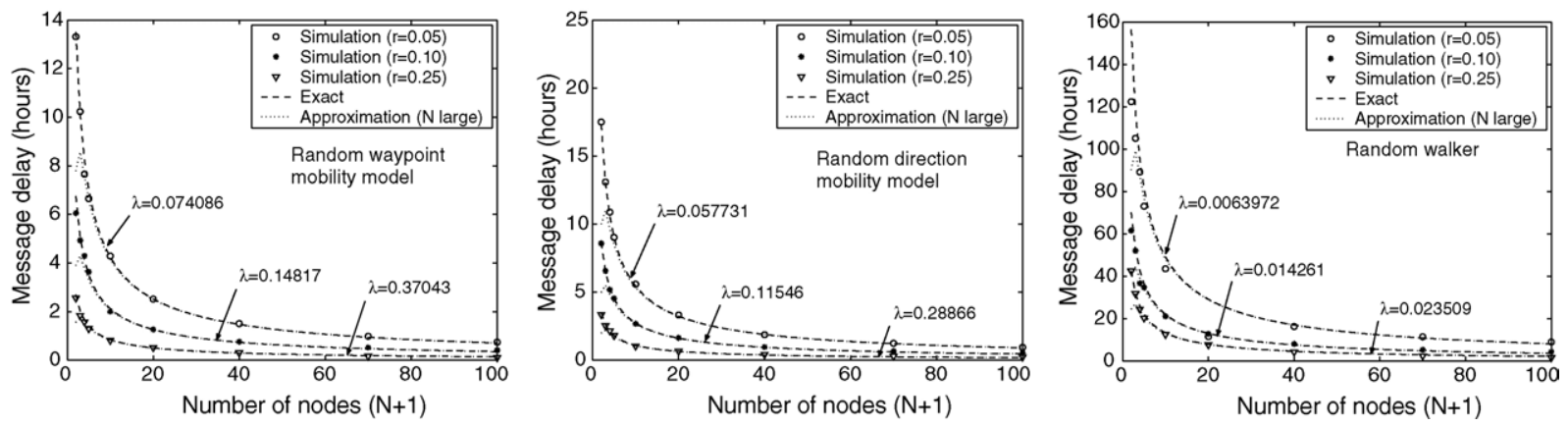

Fig. 7. Message delay vs. number of nodes: the unrestricted multicopy protocol.

\subsection{Distribution of the number of copies}

Figs. 8-10 compare the distribution of the number of copies at message delivery time obtained through simulations (represented by bars) and by the analytical model (solid lines), under both relay protocols and for 40 nodes (i.e. $N=39$ ). Results for the two-hop multicopy protocol are displayed in Figs. 8 and 9 for the random waypoint and the random walker mobility models, respectively (results for the random direction mobility models are identical to that of the random waypoint mobility model and have not been displayed). We observe that for all three mobility models the fit is quite good when $r=50 \mathrm{~m}$ and that it
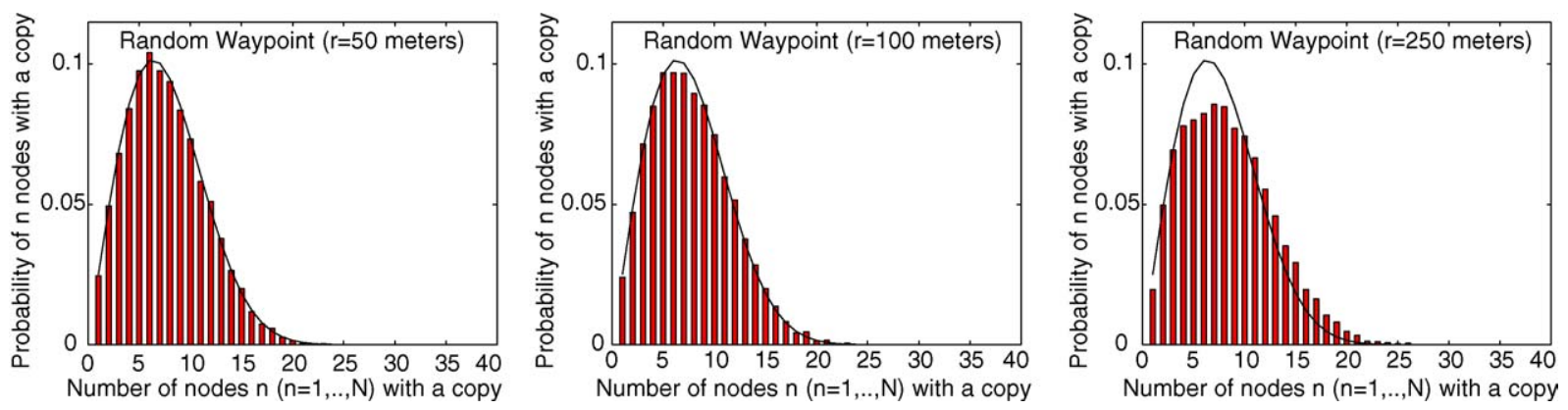

Fig. 8. Distribution number of copies: the two-hop multicopy protocol under the random waypoint model.
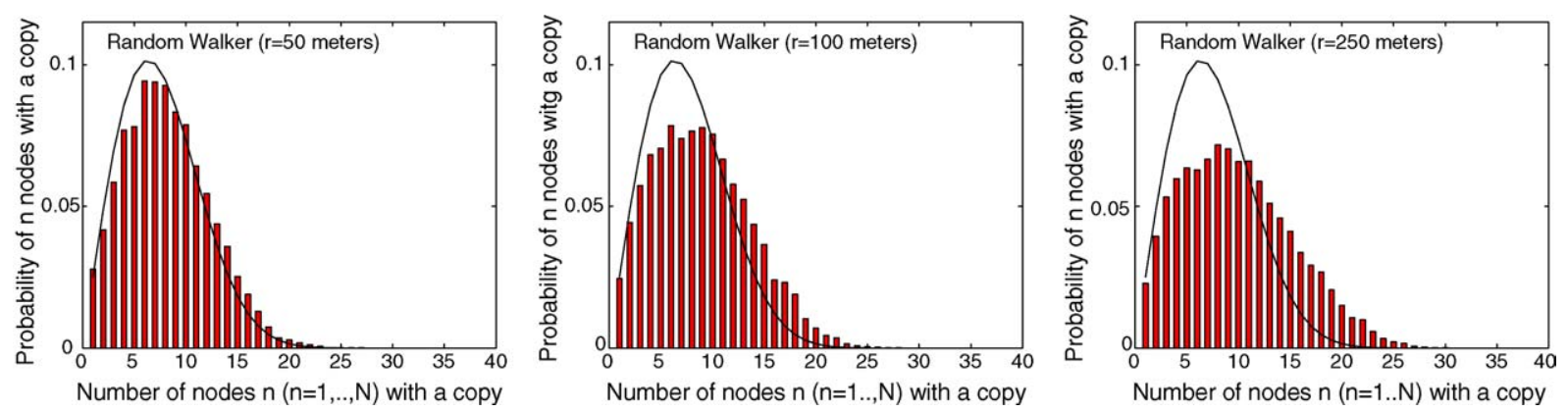

Fig. 9. Distribution of the number of copies: the two-hop multicopy protocol under the random walker model. 

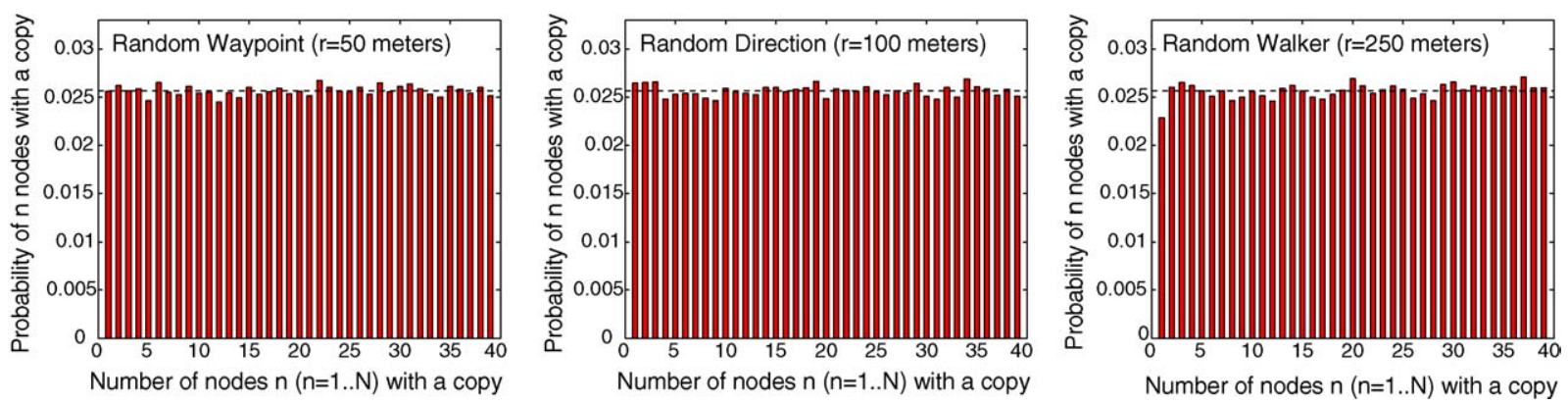

Fig. 10. Distribution of the number of copies: the unrestricted multicopy protocol.

deteriorates as $r$ increases (although the results are still acceptable for $r=100 \mathrm{~m}$ for the random waypoint mobility model and the random direction mobility model).

Results for the unrestricted multicopy protocol are reported in Fig. 10. Recall that for this protocol the number of copies is uniformly distributed in the analytical model, namely, $P\left(N_{\mathrm{U}}=i\right)=1 / 39 \approx 0.0256$ for all $i=1, \ldots, 39$ (see Proposition 1). Results are displayed for each mobility model, each for a different transmission range. We can see that in all cases the distribution of the number of copies is very close to the uniform distribution.

These results give a good indication that our model, despite its genericness, is able to capture the main features of the interaction of the mobility models and the relay protocols.

\section{A transmission range which depends on the number of nodes}

We have observed in Section 3 that, for the three mobility models considered in this chapter, the intermeeting time intensity $(\lambda)$ is well approximated by a linear function of the transmission range $r$. This approximation is valid as long as $r$ is not "too large" with respect to the size of the area in which the nodes move. On the other hand, when the number of nodes increases $r$ should decrease to prevent interferences from becoming excessive. Putting these two observations together yields $\lambda=\mathcal{O}(r(N))$, where $r(N)$, the transmission range for a network with $N$ nodes, is a decreasing function of $N$. Introducing this behaviour of $\lambda$ into Theorem 2 immediately gives the following:

Corollary 5. If $\lambda$ is linearly dependent on the radius and the radius $r(N)$ is dependent on the number of nodes, then for large $N$

$$
\mathbb{E}\left[T_{2}\right]=\mathcal{O}\left(\frac{1}{\sqrt{N} r(N)}\right) \quad \text { and } \quad \mathbb{E}\left[T_{U}\right]=\mathcal{O}\left(\frac{\log (N)}{\operatorname{Nr}(N)}\right) .
$$

If we choose $r(N)=\mathcal{O}(1 / \sqrt{N})$ in order to keep interference at an acceptable level (it is shown in [7] [14, Lemma 1] that $r(N)=\mathcal{O}(1 / \sqrt{N})$ achieves a constant capacity per node with the two-hop relay protocol), then

$$
\mathbb{E}\left[T_{2}\right]=\mathcal{O}(1) \quad \text { and } \quad \mathbb{E}\left[T_{U}\right]=\mathcal{O}\left(\frac{\log (N)}{\sqrt{N}}\right) .
$$


Alternatively, one may wish to find the function $r(N)$ so that the expected message delay under the unrestricted multicopy protocol is $\mathcal{O}(1)$ as the number of nodes becomes large. This is achieved when $r(N)=\mathcal{O}(\log (N) / N)$ leading to $\mathbb{E}\left[T_{2}\right]=\mathcal{O}(\sqrt{N} / \log (N))$.

Remark 6. Let $T_{R}$ be the message delay under the two-hop relay protocol. Recall that in this protocol a message is relayed instead of copied [7,14,15]. Similar to the analysis conducted in Section 2, it can be shown that $\mathbb{E}\left[T_{R}\right]=\lambda^{-1}\left(1+1 / N-1 / N^{2}\right)=\lambda^{-1}(1+\mathcal{O}(1 / N))$. Since $\lambda=\mathcal{O}(r(N))$ for the random waypoint model, and with the scaling $r=\mathcal{O}(1 / \sqrt{N})$, we find that the expected message delay under the two-hop relay protocol is $\mathcal{O}(\sqrt{N})$, just as was found in [14] but for nodes moving on a sphere. ${ }^{2}$

\section{Concluding remarks}

In this paper we have introduced a simple stochastic model with only two parameters to characterize the delay incurred by a message in a mobile ad hoc network. The LST of the message delay, and the distribution of the number of copies of the message at the time the message is delivered to the destination node, have been derived for two protocols: the two-hop and the unrestricted multicopy protocol. These analytical results have been compared to simulation results obtained for three different mobility models: the random waypoint, the random direction and the random walker mobility models. For small to moderate transmission radii (with respect to the size of the area), the analytical results very closely match the simulation results.

We believe that this generic model can be used to evaluate and compare the performance of different routing protocols for MANET for a wide range of mobility models.

Future research will focus on the message delivery within a certain timeframe, the inclusion of queueing delays, non-homogeneous scenarios (nodes have a different or a changing transmission range), the inclusion of interference and transmission times, and the study of other mobility models (two-dimensional, three-dimensional, or on a sphere).

\section{Appendix A. Proof of the asymptotic expansion of Eq. (12)}

In this appendix it will be shown that (for large $N$ )

$$
\sum_{i=1}^{N} \frac{i^{2} N !}{(N-i) ! N^{i}}=N^{3 / 2} \sqrt{\frac{\pi}{2}}+\mathcal{O}(N) .
$$

Proof. Define $A(N):=\sum_{i=1}^{N} \frac{i^{2} N !}{(N-i) ! N^{i}}$. If it were not for the presence of the factor $i^{2}$ in $A(N)$, then this quantity would be the Ramanujan Q-distribution [13, page 188], which is also known as the birthday function and often shows up in the analysis of algorithms.

The derivation of the approximation (A.1) follows that of the Ramanujan Q-distribution approximation [13, Proposition 4.8]. We now outline it.

\footnotetext{
2 The Brownian motion mobility model was studied in [15], whereas in this paper we consider the random walker mobility model. To go from the latter movement to the former care must be taken in terms of the limit and the metric under consideration (expected delay).
} 
Let $i_{0}:=\left\lfloor N^{3 / 5}\right\rfloor$. This implies that $i_{0}^{2} / N \rightarrow \infty$ as $N \rightarrow \infty$ and $i_{0}=\mathrm{o}\left(N^{2 / 3}\right)$. We have

$$
A(N)=\sum_{i=1}^{i_{0}} \frac{i^{2} N !}{(N-i) ! N^{i}}+B(N)
$$

with $B(N):=\sum_{i=i_{0}+1}^{N} \frac{i^{2} N !}{(N-i) ! N^{i}}$.

$B(N)$ is an exponentially small function of $N$, in the sense that $B(N)$ is $\mathcal{O}\left(1 / N^{a}\right)$ for any $a>0$. The proof of this result goes as follows. It is shown in the proof of Proposition 4.8 in [13] that $C(N):=$ $\sum_{i=i_{0}+1}^{N} \frac{N !}{(N-i) ! N^{i}}$ is an exponentially small quantity. On the other hand, $B(N) \leq N^{2} C(N)$, from which we conclude that $B(N)$ is exponentially small since the product of an exponentially small quantity and any polynomial in $N$ remains an exponentially small quantity [13, Exercise 4.10, p. 158].

Therefore, $A(N)=\sum_{i=1}^{i_{0}} \frac{i^{2} N !}{(N-i) ! N^{i}}+\Delta(N)$, where $\Delta(N)$ represents a function which is exponentially small. For any integer $i$ that is $\mathrm{o}\left(N^{2 / 3}\right)$ it is shown in [13, Proposition 4.4] that

$$
\frac{N !}{(N-i) ! N^{i}}=\mathrm{e}^{-i^{2} /(2 N)}\left(1+\mathcal{O}\left(\frac{i}{N}\right)+\mathcal{O}\left(\frac{i^{3}}{N^{2}}\right)\right) .
$$

Since $i=\mathrm{o}\left(N^{2 / 3}\right)$ whenever $1 \leq i \leq i_{0}$, we deduce from (A.2) that

$$
A(N)=\sum_{i=1}^{i_{0}} i^{2} \mathrm{e}^{-i^{2} /(2 N)}\left(1+\mathcal{O}\left(\frac{i}{N}\right)+\mathcal{O}\left(\frac{i^{3}}{N^{2}}\right)\right)+\Delta(N)
$$

By applying the Euler-MacLaurin summation [13, Proposition 4.2] to the functions $x^{3} \mathrm{e}^{-x^{2} / 2}$ and $x^{5} \mathrm{e}^{-x^{2} / 2}$ we find (see [13, Exercice 4.9] for similar results)

$$
\sum_{i=1}^{i_{0}} i^{2} \mathrm{e}^{-i^{2} /(2 N)} \mathcal{O}\left(\frac{i}{N}\right)=\mathcal{O}(N) \quad \text { and } \quad \sum_{i=1}^{i_{0}} i^{2} \mathrm{e}^{-i^{2} /(2 N)} \mathcal{O}\left(\frac{i^{3}}{N^{2}}\right)=\mathcal{O}(N)
$$

respectively. Hence, $A(N)=\sum_{i=1}^{i_{0}} i^{2} \mathrm{e}^{-i^{2} /(2 N)}+\mathcal{O}(N)$. By noting that $i^{2} \mathrm{e}^{-i^{2} /(2 N)}$ is exponentially small for $i>i_{0}$, we can add all terms for $i>i_{0}$ into the summation to give

$$
A(N)=\sum_{i \geq 1} i^{2} \mathrm{e}^{-i^{2} /(2 N)}+\mathcal{O}(N)
$$

The above summation is the summation of the function $N x^{2} \mathrm{e}^{-x^{2} / 2}$ at regularly spaced points with step $1 / \sqrt{N}$. Another application of the Euler-MacLaurin formula [13, Proposition 4.2] yields

$$
\sum_{i \geq 1} i^{2} \mathrm{e}^{-i^{2} /(2 N)}=N^{3 / 2} \int_{0}^{\infty} x^{2} \mathrm{e}^{-x^{2} / 2} \mathrm{~d} x+\mathcal{O}(N)=N^{3 / 2} \sqrt{\frac{\pi}{2}}+\mathcal{O}(N),
$$

so that $A(N)=N^{3 / 2} \sqrt{\frac{\pi}{2}}+\mathcal{O}(N)$ from (A.3) and (A.4), which concludes the proof of the lemma. 


\section{References}

[1] C. Bettstetter, Mobility modeling in wireless networks: categorization, smooth movement, and border effects, ACM Mobile Comput. Commun. Rev. 5 (3) (2001) 55-67.

[2] C. Bettstetter, C. Wagner, The spatial node distribution of the random waypoint mobility model, in: Proceedings of the First German Workshop Mobile Ad hoc Networks (WMAN), 2002, pp. 41-58.

[3] J. Broch, A.D. Maltz, B.D. Johnson, Y.-C. Hu, J. Jetcheva, A performance comparison of multi-hop wireless ad hoc network routing protocols, in: Proceedings of the ACM International Conference on Mobile Computing and Networks (ACM MOBICOM), Dallas, TX, October 25-30, 1998, pp. 85-97.

[4] W. Feller, An Introduction to Probability Theory and its Applications, John Wiley and Sons, Inc., 1950.

[5] R. Groenevelt, Stochastic Models for Ad hoc Networks, PhD thesis, INRIA, April, 2005.

[6] R. Groenevelt, E. Altman, P. Nain, Relaying in Mobile Ad hoc Networks, In: Proceedings of the WiOpt'04, UK, March, 2004.

[7] M. Grossglauser, D. Tse, Mobility increases the capacity of ad hoc wireless networks, ACM/IEEE Trans. Network. 10 (4) (2002) 477-486.

[8] R. Guérin, Channel occupancy time distribution in a cellular radio system, IEEE Trans. Vehicular Technol. 36 (1987) 89-99.

[9] Q. Li, D. Rus, Sending messages to mobile users in disconnected ad hoc wireless networks, in: Proceedings of the Sixth ACM MobiCom, Boston, MA, August 6-11, 2000.

[10] D. Nain, N. Petigara, H. Bakakrishnan, Integrated routing and storage for messaging applications in mobile ad hoc networks, Mobile Networks Appl. (MONET) 9 (6) (2004) 595-604.

[11] P. Nain, D. Towsley, B. Liu, Z. Liu, Properties of Random Direction Models, in: Proceedings of the IEEE Infocom 2005, Miami, FL, March 13-17, 2005.

[12] W. Navidi, T. Camp, Stationary distributions for the random waypoint mobility model, IEEE Trans. Mobile Comput. 3 (1) (2004) 99-108.

[13] R. Sedgewick, P. Flajolet, An Introduction to the Analysis of Algorithms, Addison-Wiley, 1996.

[14] G. Sharma, R.R. Mazumdar, Delay and capacity trade-offs for wireless ad hoc networks with random mobility, Submitted for publication.

[15] G. Sharma, R.R. Mazumdar, Scaling laws for capacity and delay in wireless ad hoc networks with random mobility, in: Proceedings of the ICC, Paris, France, June 20-24, 2004.

[16] J. Yoon, M. Liu, B. Noble, Random Waypoint Considered Harmful, in: Proceedings of the IEEE Infocom, San Francisco, April 1-3, 2003, pp. 1312-1321.

[17] W. Zhao, M. Ammar, Message ferrying: proactive routing in highly-partioned wireless ad hoc networks, in: Proceedings of the Ninth IEEE Workshop on FTDCS'03, San Juan, Puerto Rico, May, 28-30, 2003.

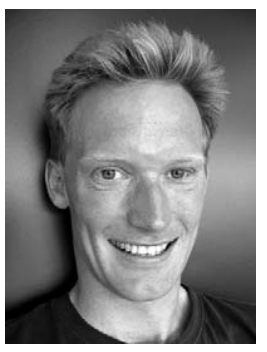

Robin Groenevelt received a MSc in Business Mathematics and Computer Science (1998) and a MSc in Mathematics (2000) from the Vrije Universiteit, Amsterdam. From 1999 to 2001 he worked as a consultant in the Center for Applied Mathematics at the Rabobank in the Netherlands. In 2005 he obtained his PhD in Computer Science at INRIA (Sophia Antipolis), where he worked on the modeling and performance analysis of mobile ad hoc networks. In 2004-2005 he held a teaching/research position at the University of Nice-Sophia Antipolis. Since September 2005 he works as a researcher in the R\&D Techlabs of Accenture in Sophia Antipolis, France.

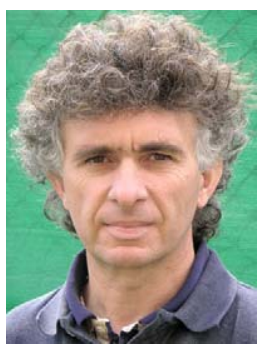

Philippe Nain received the Maitrise Es-Sciences in Mathematics in 1978, the Diplôme d'Etudes Approfondies in Statistics in 1979, and the Doctorat de 3ème cycle specializing in Modeling of Computer Systems in 1981 from the University of Paris XI, Orsay, France. In 1987 he received the Doctorat d'Etat in Applied Mathematics from the University Pierre and Marie Curie, Paris, France. Since december 1981 he has been with INRIA where he is currently the head of the research project Maestro devoted to the modeling of computer systems and telecommunications networks. He has held visiting appointments at the University of Massachusetts (1993-1994), at the University of Maryland (1987) and at North Carolina State University (1988). His research interests include modeling and performance evaluation of communication networks. He is an Associate Editor of Performance Evaluation and Operations Research Letters, 
and was an Associate Editor of IEEE Transactions on Automatic Control. He was a co-program chair of the ACM Sigmetrics 2000 conference, the general chair of the Performance 2005 conference, and he is a member of IFIP WG 7.3.

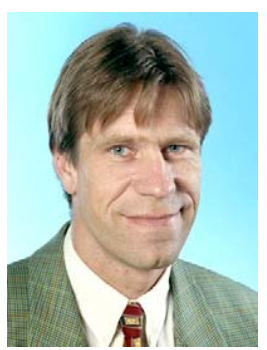

Ger Koole is a professor in the Department of Mathematics at the Vrije Universiteit Amsterdam. He graduated in 1992 from Leiden University, and held a postdoc position at CWI (Amsterdam) and INRIA (Sophia Antipolis). He is interested in the theory and applications of controlled queueing systems. His email address is koole@few.vu.nl, and his web page is http://www.math.vu.nl/ koole. 